\title{
A Stochastic Geometry Approach to Transmission Capacity in Wireless Cooperative Networks
}

\author{
Zhengguo Sheng*, D. L. Goeckel\$, Kin K Leung*, Zhiguo Ding† \\ *Department of Electrical and Electronic Engineering, Imperial College, UK \\ $\ddagger$ Electrical Department, University of Massachusetts, Amherst, MA, USA \\ $\dagger$ Department of Communication Systems, Lancaster University, UK \\ Email: zhengguo.sheng06@imperial.ac.uk
}

\begin{abstract}
In this paper, we employ a stochastic geometry model to analyze transmission capacity in wireless cooperative networks. Assuming that simultaneous transmitters are randomly located in space according to Poisson point process with density $\rho$, we develop the bound performances on outage probability and outage capacity for both direct transmission and Decodeand-Forward (DAF) cooperative scheme. Due to the nature of multipath propagation of cooperative transmission, we define regional capacity as the multiplied product of average density of successful simultaneous transmissions, achieved outage capacity and transmission distance. It shows that the regional capacity for cooperative transmission scales as $\Theta(\sqrt{\rho})$, which is the same as the transport capacity for wireless network. Furthermore, Monte Carlo simulations demonstrate the significant improvement on the transmission capacity by using cooperative transmission.
\end{abstract}

\section{INTRODUCTION}

Cooperative transmission (CT) has recently attracted much attention as an effective technique to combat multi-path fading and enhance receiver reliability in wireless communication systems. The key feature of cooperative transmission is to encourage single-antenna devices to cooperatively share their antennas such that a virtual antenna array can be constructed, thereby, enabling performance gains to be significantly boosted. However, existing work more focuses on reception reliability (i.e., BER) and energy issues [1]-[3]; the potential of transmission capacity of cooperative networks has not been fully explored.

In our previous work [4], we have analyzed the network throughput for wireless cooperative networks in a regular linear network scenario. Although there are some state-of-art works on ad hoc network capacity [5]-[7], this fundamental problem for a general cooperative network is still unclear. In this paper, we approach this problem through a stochastic geometry model [6] which employs a homogeneous Poisson point process to represent the distribution of simultaneous transmitters.

We use a simple propagation model that only includes distance dependent path-loss and co-channel interferences [8], where the interference behavior is clearly presented by the

This research was sponsored by US Army Research laboratory and the UK Ministry of Defence and was accomplished under Agreement Number W911NF-06-3-0001. The views and conclusions contained in this document are those of the authors and should not be interpreted as representing the official policies, either expressed or implied, of the US Army Research Laboratory, the U.S. Government, the UK Ministry of Defense, or the UK Government. The US and UK Governments are authorized to reproduce and distribute reprints for Government purposes notwithstanding any copyright notation hereon. stochastic geometry model. In order to evaluate the performance of a more practical distributed ad hoc networks, we assume that all the transmitters are using ALOHA-type [9] transmission without centralized scheduling. We only consider the single hop transmission, whereas the multi-hop case is not directly considered (although our results can be extended to more general cases with multiple hops).

The contribution of this paper is three-fold: first, we investigate the average interference performance through the stochastic geometry modeling and then use it to obtain the upper bounds on outage probability for both direct transmission and Decode-and Forward (DAF) cooperation scheme. The outage event is decided by the received signal-to-noise-plusinterference (SINR) ratio at the receiver. Such bounds can then be manipulated to derive the lower bounds on the outage capacity for both schemes. Second, consider the broadcast nature of cooperative transmission that multipath propagations within a region are accumulated at a receiver, we define regional capacity in $\mathrm{bit} / \mathrm{s} / \mathrm{Hz} / \mathrm{m}$ as the multiplied product of average density of successful simultaneous transmissions, achieved outage capacity and transmission distance. As a result, Monte Carlo simulations demonstrate the significant improvement on the transmission capacity by using cooperative transmission. Third, compared with transport capacity [5], we further show that the regional capacity for cooperative transmission is also scaled as $\Theta(\sqrt{\rho})$, where $\rho$ is the density of simultaneous transmitters. Overall, cooperative transmission has larger regional capacity than direct transmission, but still achieves the same scaling result of transport capacity.

The remainder of this paper is organized as follows. In Section II, we describe the system model. Specifically, we formulate the average interference using stochastic approach as well as the outage behaviors for both direct and cooperation schemes. In Section III, we introduce the regional capacity. In Section IV, we provide some numerical results to further show the performance gain of cooperative transmission on regional capacity and the paper concludes in Section V.

\section{SySTEM MODEL}

We consider an infinitely dense wireless network which allows the selection of node at any location. All the simultaneous transmitters within the network use the same transmission power $P_{t}$ to transmit over the same distance $d$ and $P_{n}$ is the noise power. We employ a propagation model to only consider distance dependent path-loss and co-channel interferences. 
The wireless link between the nodes $i$ and $j$ is modeled as $\mathrm{a}_{i j}=d_{i j}^{-\alpha / 2}$, where $d_{i j}$ is the distance between the nodes $i$ and $j$, represents the large-scale behavior of the channel gain and $\alpha$ is the path-loss exponent.

\section{A. Interference Model}

The simultaneous transmitters are modeled by homogeneous Poisson point process in the network with density $\rho$ and the transmitters are using a fixed transmission power $P_{t}$ to transmit. Given that a receiver is located at origin $(0,0)$ and the distance between each transmitter and the origin is $X_{i}$, the accumulated interference at the origin is

$$
\mathrm{I}=\sum_{i \in \Phi} P_{t}\left|X_{i}\right|^{-\alpha}
$$

where $\Phi$ is the set of simultaneous transmitters and $\alpha$ is path loss exponent. The probability distribution function of interference can be expressed as

$$
P(\mathrm{I}<x)=\sum_{n=0}^{\infty} P\left(\mathrm{I}_{n}<x \mid n\right) P(n),
$$

Since we can bound the tail of the distribution using moments, the mean and variance of I can be derived as

$$
\begin{aligned}
E[\mathrm{I}] & =\frac{2 \pi P_{t} \rho s^{2-\alpha}}{\alpha-2} \\
\operatorname{Var}[\mathrm{I}] & =\frac{\pi P_{t}^{2} \rho s^{2(1-\alpha)}}{\alpha-1} .
\end{aligned}
$$

where $s$ is the minimum far-field ${ }^{1}$ distance to the origin and we only consider the case of $\alpha>2$. The development of (3) and (4) is provided in Appendix A.

\section{B. Outage Behavior of Direct Transmission}

To establish baseline performance under direct transmission, the maximum average mutual information between the source and the destination in this network scenario is given by

$$
\begin{aligned}
\mathrm{I}_{D} & =\log \left(1+\operatorname{SINR}_{s, d}\right) \\
& =\log \left(1+\frac{P_{t} d_{s, d}^{-\alpha}}{P_{n}+\sum_{i \in \Phi} P_{t}\left|X_{i}\right|^{-\alpha}}\right)
\end{aligned}
$$

\footnotetext{
${ }^{1}$ The path-loss model is based on a far-field assumption: the distance is assumed to be much larger than the carrier wavelength. When the distance is of the order or shorter than the carrier wavelength, the simple path-loss model obviously does not hold anymore as path loss can potentially become path gain. The reason is that near-field electromagnetics now come into play.
}

The outage event for a desired transmission data rate $R$ in bit/s/Hz is given by $\mathrm{I}_{D}<R$ and the outage probability satisfies

$$
\begin{aligned}
& \operatorname{Pr}\left[\mathrm{I}_{D}<R\right]=\operatorname{Pr}\left[\frac{P_{t} d_{s, d}^{-\alpha}}{P_{n}+\sum_{i \in \Phi} P_{t}\left|X_{i}\right|^{-\alpha}} \leq 2^{R}-1\right] \\
& =\operatorname{Pr}\left[\frac{d_{s, d}^{-\alpha}}{\frac{P_{n}}{P_{t}}+\sum_{i \in \Phi}\left|X_{i}\right|^{-\alpha}} \leq 2^{R}-1\right] \\
& =\operatorname{Pr}\left[\frac{P_{n}}{P_{t}}+\sum_{i \in \Phi}\left|X_{i}\right|^{-\alpha} \geq \frac{d_{s, d}^{-\alpha}}{2^{R}-1}\right] \\
& =\operatorname{Pr}\left[\sum_{i \in \Phi}\left|X_{i}\right|^{-\alpha} \geq \frac{d_{s, d}^{-\alpha}}{2^{R}-1}-\frac{P_{n}}{P_{t}}\right]
\end{aligned}
$$

Using the Chebyshev bound, the upper bound performance of (6) can be derived as

$$
\begin{aligned}
& =\operatorname{Pr}\left[\sum_{i \in \Phi}\left|X_{i}\right|^{-\alpha}-E[\mathrm{I}] \geq \frac{d_{s, d}^{-\alpha}}{2^{R}-1}-\frac{P_{n}}{P_{t}}-E[\mathrm{I}]\right] \\
& \leq \operatorname{Pr}\left[\left.\left|\sum_{i \in \Phi}\right| X_{i}\right|^{-\alpha}-E[\mathrm{I}] \mid \geq \epsilon\right] \\
& \leq \frac{\operatorname{Var}[\mathrm{I}]}{\epsilon^{2}}
\end{aligned}
$$

where $\epsilon=\frac{d_{s, d}^{-\alpha}}{2^{R}-1}-\frac{P_{n}}{P_{t}}-E[\mathrm{I}], E[\mathrm{I}]$ and $\operatorname{Var}[\mathrm{I}]$ can be found from (3) and (4). The outage probability of direct transmission is

$$
p_{D T}^{\text {out }} \leq \frac{\pi P_{t}^{2} \rho s^{2(1-\alpha)}}{(\alpha-1)\left(\frac{d_{s, d}^{-\alpha}}{2^{R}-1}-\frac{P_{n}}{P_{t}}-\frac{2 \pi P_{t} \rho s^{2-\alpha}}{\alpha-2}\right)^{2}} .
$$

\section{Outage Behavior of Cooperative Transmission}

As compared with direct transmission, the Decode-andForward (DAF) allows the relay to decode the signals from the source, re-encode and retransmit the signals to the destination. Specifically, here we consider the Selection Decode-Forward [2] transmission. If the relay node cannot successfully decode the signals from the source, the source simply repeats its transmission directly to the destination; otherwise, the relay forwards what it received from the source using decodeforward.

Let $d_{s, d}, d_{s, r}$ and $d_{r, d}$ be the respective distances among the source, relay and destination. During the first time slot, the destination receives $y_{d}=\frac{x_{s}}{d_{s, d}^{\alpha / 2}}+n_{d}$ from the source node, where $x_{s}$ is the information transmitted by the source and $n_{d}$ is white noise. During the second time slot, the destination node receives

$$
y_{d}= \begin{cases}\frac{x_{s}}{d_{s, d}^{\alpha / 2}}+n_{d}, & \text { if } \operatorname{SINR}_{s, r}<q(R) \\ \frac{x_{r}}{d_{r, d}^{\alpha / 2}}+n_{d}, & \text { if } \operatorname{SINR}_{s, r} \geq q(R)\end{cases}
$$

where $q(R)=\left(2^{2 R}-1\right)$ can be derived from direct transmission and is analogous to (5). As can be seen from the first condition of (9), when the link between the source and the relay is so poor that the relay is not able to decode, there is no diversity gain can be achieved and the source is repeating its transmission during this slot. The second 
condition corresponds to the case when the relay can decode and repeat the source transmission, thus obtaining the secondorder diversity gain through CT. Therefore, choosing a proper relay to guarantee the link quality is critical in achieving good system performance.

Assume that a relay node is randomly selected. Hence the mutual information of this cooperative link can be shown as

$$
\mathrm{I}_{C}= \begin{cases}\frac{1}{2} \log \left(1+2 \mathrm{SINR}_{s, d}\right), & \operatorname{SINR}_{s, r}<q(R) \\ \frac{1}{2} \log \left(1+\operatorname{SINR}_{s, d}+\operatorname{SINR}_{r, d}\right), & \operatorname{SINR}_{s, r} \geq q(R)\end{cases}
$$

Therefore, the outage event for the Selected Decode-andForward (SDF) is given by $\mathrm{I}_{C}<R$ and is equivalent to the event

$$
\begin{aligned}
& \left(\left\{\operatorname{SINR}_{s, r}<q(R)\right\} \cap\left\{2 \operatorname{SINR}_{s, d}<q(R)\right\}\right) \\
& \cup\left(\left\{\operatorname{SINR}_{s, r} \geq q(R)\right\} \cap\left\{\operatorname{SINR}_{s, d}+\operatorname{SINR}_{r, d}<q(R)\right\}\right),
\end{aligned}
$$

As we can see above, two events of the union in (11) corresponds to two cases in (10), respectively. Because the events in union of (11) are mutually exclusive, the outage probability becomes a sum

$$
\begin{aligned}
& p_{C T}^{\text {out }}=\operatorname{Pr}\left[\mathbf{I}_{C}<R\right] \\
& =\underbrace{\operatorname{Pr}\left[\operatorname{SINR}_{s, r}<q(R)\right]}_{A} \underbrace{\operatorname{Pr}\left[2 \operatorname{SINR}_{s, d}<q(R)\right]}_{B} \\
& +\underbrace{\operatorname{Pr}\left[\operatorname{SINR}_{s, r} \geq q(R)\right]}_{C} \underbrace{\operatorname{Pr}\left[\operatorname{SINR}_{s, d}+\operatorname{SINR}_{r, d}<q(R)\right]}_{D}
\end{aligned}
$$

By computing each component in (12), we have

$$
\begin{aligned}
& A \leq \frac{\pi P_{t}^{2} \rho s^{2(1-\alpha)}}{(\alpha-1)\left(\frac{d_{s, r}^{-\alpha}}{2^{2 R}-1}-\frac{P_{n}}{P_{t}}-\frac{2 \pi P_{t} \rho s^{2-\alpha}}{\alpha-2}\right)^{2}} \\
& B \leq \frac{\pi P_{t}^{2} \rho s^{2(1-\alpha)}}{(\alpha-1)\left(\frac{2 d_{s, d}^{-\alpha}}{2^{2 R}-1}-\frac{P_{n}}{P_{t}}-\frac{2 \pi P_{t} \rho s^{2-\alpha}}{\alpha-2}\right)^{2}} \\
& C \rightarrow 1
\end{aligned}
$$

when keeping the dominant term under the condition that outage probability $A$ is small. Since the whole transmission follows a same random process regard to a fixed receiver in two time slots, we assume the interference is statistically the same, $D$ can be derived as

$$
\begin{aligned}
& D=\operatorname{Pr}\left[\frac{P_{t}\left(d_{s, d}^{-\alpha}+d_{r, d}^{-\alpha}\right)}{P_{n}+\sum_{i \in \Phi} P_{t}\left|X_{i}\right|^{-\alpha}} \leq 2^{2 R}-1\right] \\
& \leq \frac{\pi P_{t}^{2} \rho s^{2(1-\alpha)}}{(\alpha-1)\left(\frac{d_{s, d}^{-\alpha}+d_{r, d}^{-\alpha}}{2^{2 R}-1}-\frac{P_{n}}{P_{t}}-\frac{2 \pi P_{t} \rho s^{2-\alpha}}{\alpha-2}\right)^{2}}
\end{aligned}
$$

Finally, we obtain a closed form expression for (12).

$$
\begin{aligned}
& p_{C T}^{\text {out }} \leq \frac{\pi P_{t}^{2} \rho s^{2(1-\alpha)}}{(\alpha-1)\left(\frac{d_{s, d}^{-\alpha}+d_{r, d}^{-\alpha}}{2^{2 R}-1}-\frac{P_{n}}{P_{t}}-E[\mathrm{I}]\right)^{2}}+ \\
& \frac{\left(\pi P_{t}^{2} \rho s^{2(1-\alpha)}\right)^{2}}{(\alpha-1)^{2}\left(\frac{d_{s, r}^{-\alpha}}{2^{2 R}-1}-\frac{P_{n}}{P_{t}}-E[\mathrm{I}]\right)^{2}\left(\frac{2 d_{s, d}^{-\alpha}}{2^{2 R}-1}-\frac{P_{n}}{P_{t}}-E[\mathrm{I}]\right)^{2}}
\end{aligned}
$$

\section{Regional CAPACITY}

Given a set of simultaneous transmissions with density $\rho$ taking place over $1 \mathrm{~m}^{2}$, we define the reginal capacity in $\mathrm{bit} / \mathrm{s} / \mathrm{Hz} / \mathrm{m}$ when the whole network transmits one bit/s/Hz to its destination in a distance of one meter. The definition can be express as

$$
\lambda=p_{\text {out }}^{-1}(\varepsilon)(1-\varepsilon) \rho d .
$$

where $p_{\text {out }}^{-1}(\varepsilon)$ is the outage capacity in bit/s/Hz, $\varepsilon$ is a target error rate, $\rho$ is the number of active links $/ \mathrm{m}^{2}$ and $d$ is the average transmission distance.

Since we have the upper bounds on outage probability for both direct transmission and cooperative transmission, the regional capacity for each scheme can be derived as following:

\section{A. For Direct Transmission}

According to (8), the lower bound outage capacity is

$$
p_{\text {out }}^{-1}(\varepsilon) \geq \log \left(\frac{d_{s, d}^{-\alpha}}{\sqrt{\frac{P_{t}^{2} \pi \rho s^{2(1-\alpha)}}{(\alpha-1) \varepsilon}}+\frac{P_{n}}{P_{t}}+\frac{2 \pi P_{t} \rho s^{2-\alpha}}{\alpha-2}}+1\right),
$$

We assume that all transmissions are over the same distance of $d$ meters. Hence, the regional capacity of direct transmission is

$$
\lambda_{D T} \geq \log \left(\frac{d^{-\alpha}}{\sqrt{\frac{P_{t}^{2} \pi \rho s^{2(1-\alpha)}}{(\alpha-1) \varepsilon}}+\frac{P_{n}}{P_{t}}+E[\mathbf{I}]}+1\right)(1-\epsilon) \rho d .
$$

\section{B. For Cooperative Transmission}

Since the transmission distance between the source and destination is $d$ meters, (13) can be further simplified as

$$
\begin{aligned}
& p_{C T}^{\text {out }} \leq \frac{\pi P_{t}^{2} \rho s^{2(1-\alpha)}}{(\alpha-1)\left(\frac{d_{r, d}^{-\alpha}+d^{-\alpha}}{2^{2 R}-1}-\frac{P_{n}}{P_{t}}-E[\mathbf{I}]\right)^{2}}+ \\
& \frac{\left(\pi P_{t}^{2} \rho s^{2(1-\alpha)}\right)^{2}}{(\alpha-1)^{2}\left(\frac{d_{s, r}^{-\alpha}}{2^{2 R}-1}-\frac{P_{n}}{P_{t}}-E[\mathbf{I}]\right)^{2}\left(\frac{2 d^{-\alpha}}{2^{2 R}-1}-\frac{P_{n}}{P_{t}}-E[\mathbf{I}]\right)^{2}} \\
& \leq \frac{\pi P_{t}^{2} \rho s^{2(1-\alpha)}}{(\alpha-1)\left(\frac{d_{r, d}^{-\alpha}+d^{-\alpha}}{2^{2 R}-1}-\frac{P_{n}}{P_{t}}-E[\mathrm{I}]\right)^{2}}+o\left(\kappa^{2}\right), \quad \text { (17) }
\end{aligned}
$$

as $\kappa=p_{D T}^{\text {out }}\left(d_{s, r}\right)$, when $d_{s, r}^{-\alpha}<2 d^{-\alpha}$; otherwise $\kappa=$ $p_{D T}^{\text {out }}\left(2^{\frac{-1}{\alpha}} d\right)$. Since the outage probability is close to $0, \kappa^{2}$ will reduce with a higher order than the first component in (17). Then the lower bound outage capacity for cooperative transmission can be approximated as

$$
p_{\text {out }}^{-1}(\varepsilon) \geq \frac{1}{2} \log \left(\frac{d_{r, d}^{-\alpha}+d^{-\alpha}}{\sqrt{\frac{P_{t}^{2} \pi \rho s^{2(1-\alpha)}}{(\alpha-1) \varepsilon}}+\frac{P_{n}}{P_{t}}+\frac{2 \pi P_{t} \rho s^{2-\alpha}}{\alpha-2}}+1\right),
$$

Therefore, the regional capacity for cooperative transmission is derived as

$$
\lambda_{C T} \geq \frac{1}{2} \log \left(\frac{d_{r, d}^{-\alpha}+d^{-\alpha}}{\sqrt{\frac{P_{t}^{2} \pi \rho s^{2(1-\alpha)}}{(\alpha-1) \varepsilon}}+\frac{P_{n}}{P_{t}}+E[\mathbf{I}]}+1\right)(1-\varepsilon) \rho d .
$$


It is worth noting that same as in transport capacity, we do not give multiple credit for the same bit broadcasted through cooperation. Based on the fact [2] that the full second-order of diversity can be achieved from DAF protocol, a higher outage capacity can be achieved through such cooperation. Its significant improvement on regional capacity which is an important criterion to measure the performance of cooperative transmission scheme will be demonstrated in next section.

\section{Comparison with Transport Capacity}

In [5], the transport capacity is defined as a multiplied product of maximum number of simultaneous transmissions (i.e., $n$ ), achieved spectral efficiency of each transmission and transmission distances. It is shown that the transport capacity can be best achieved as $\Theta(\sqrt{n})$, if we assume a normalized bandwidth of $1 \mathrm{~Hz}$.

We employ a stochastic model to measure the number of simultaneous transmissions and achievable spectral efficiency. Specifically, we assume the number of transmitters are following Poisson point process with density $\rho$. Our comparable definition for the transport capacity can be referred from (14). For cooperative transmission in an interference limited scenario, if we assume a large transmission power $P_{t} \gg P_{n}$ and we consider to use information nat ${ }^{2}$ instead of bit, then the lower bound outage capacity in (18) equals to

$$
\begin{aligned}
p_{\text {low }}^{-1}(\varepsilon)= & \frac{1}{2} \ln \left(\frac{d_{r, d}^{-\alpha}+d^{-\alpha}}{\sqrt{\frac{P_{t}^{2} \pi \rho s^{2(1-\alpha)}}{(\alpha-1) \varepsilon}}+\frac{2 \pi P_{t} \rho s^{2-\alpha}}{\alpha-2}}+1\right) \\
& \approx \frac{d_{r, d}^{-\alpha}+d^{-\alpha}}{\sqrt{\frac{P_{t}^{2} \pi \rho s^{2(1-\alpha)}}{(\alpha-1) \varepsilon}}+\frac{2 \pi P_{t} \rho s^{2-\alpha}}{\alpha-2}} \\
& \rightarrow \Theta\left(\frac{1}{\sqrt{\rho}}\right)
\end{aligned}
$$

Then, according to (19), we have the lower bound regional capacity

$$
\begin{aligned}
\lambda_{\text {low }}= & \frac{1}{2} \Theta\left(\frac{1}{\sqrt{\rho}}\right)(1-\epsilon) \rho d \\
& \rightarrow \Theta(\sqrt{\rho})
\end{aligned}
$$

as $\rho \propto n$ in our case. Therefore, it is clear that not only does the regional capacity achieve the same result of [5], but also it includes stochastic measurement for modeling simultaneous transmissions and allows more accurate computation bound for outage capacity (spectral efficiency). Furthermore, if we compare (19) with (16), depending on the relay location, cooperative transmission can obtain larger regional capacity than direct transmission, but still achieves the same scaling result of transport capacity.

\section{NUMERICAL RESULTS}

In this section, we provide some simulation results to further demonstrate the performance gain on regional capacity when

\footnotetext{
${ }^{2}$ In order to simplify notation and analysis, we use information unit nat here; 1 nat $=\log _{2}$ ebit
}

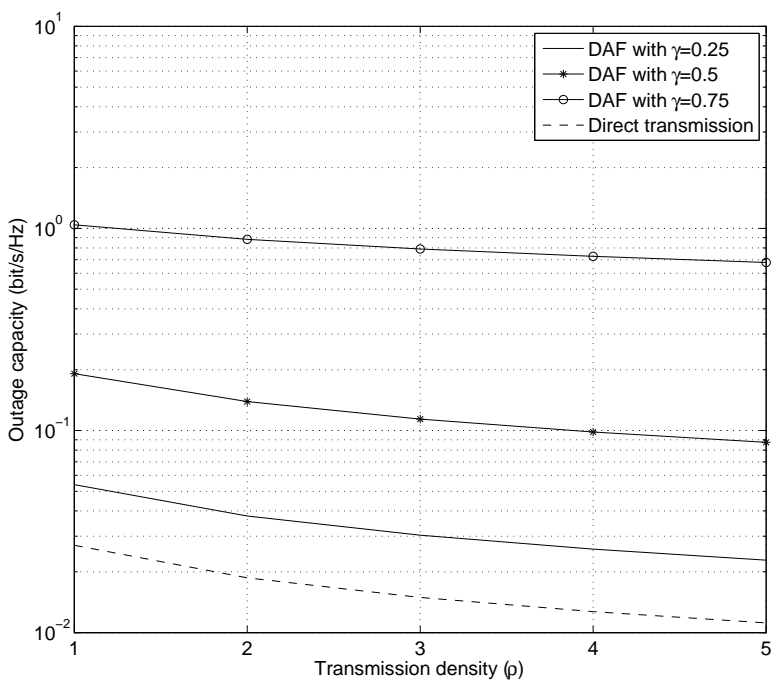

Fig. 1. Outage capacity versus transmission density

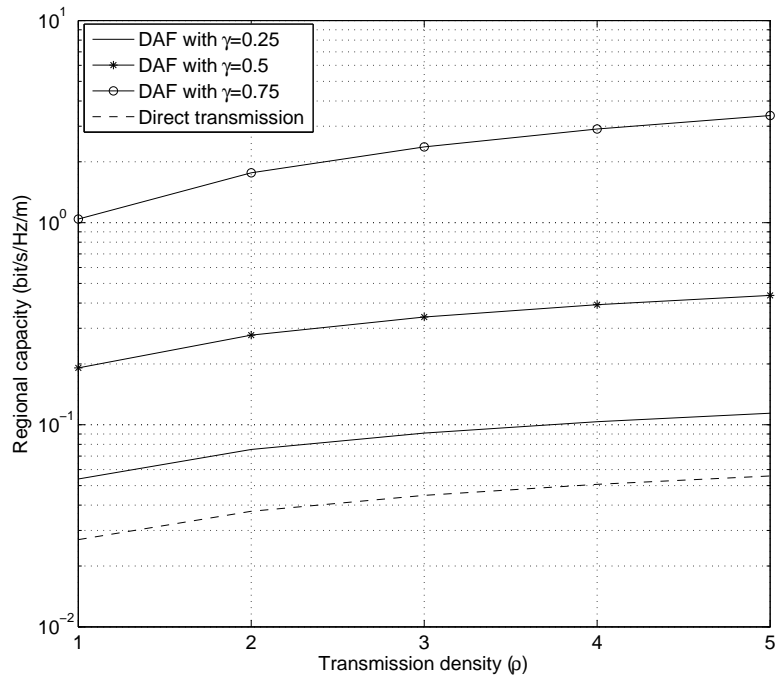

Fig. 2. Regional capacity versus transmission density

we use two different transmission schemes. We assume that the target error rate $\varepsilon=0.001$, transmission power $P_{t}=P_{n}=$ 1 , the minimum far-field distance $s=1$, path loss exponent $\alpha=4$, transmission distance between a source-destination pair is $d=1$, the relay is located on the line between the source and destination and we use $\gamma=\frac{d_{s, r}}{d_{s, d}}$ to define the normalized location of relay.

Based on different network setup, the outage capacity for both schemes shown in Fig. 1 is reduced as the transmission density increases. This is so because when the total number of simultaneous transmissions increases, average cumulative interference at each receiver will go up and thus the outage capacity will be adversely affected. Furthermore, due to the fact that the diversity gain of cooperative transmission can be significantly improved when a better located relay is se- 
lected, the outage capacity of cooperative transmission (DAF) increases as the relay closes to its destination.

By contrast, even though the outage capacity experiences decreasing when transmission density increases, the regional capacity for both schemes still shows a upper trend in Fig. 2 , since the density increases with a higher order than the decreasing order of outage capacity. This result is also consistent with the theoretical result of $\Theta(\sqrt{\rho})$. In addition, the cooperative transmission still shows much better performance than direct transmission because of the diversity gain benefit in cooperation.

\section{CONCLUSions}

We have employed a stochastic geometry model to analyze transmission capacity in wireless cooperative networks. Assuming that simultaneous transmitters are randomly located in space according to a Poisson point process with density $\rho$, we have developed the bound performances on outage probability and outage capacity for both direct transmission and Decodeand-Forward (DAF) cooperative scheme. Due to the nature of multipath propagation of cooperative transmission, we have defined regional capacity as the multiplied product of average density of successful simultaneous transmissions, achieved outage capacity and transmission distance. It has shown that the regional capacity for cooperative transmission achieves $\Theta(\sqrt{\rho})$, which is the same as the transport capacity for wireless network. Furthermore, Monte Carlo simulations have demonstrated the significant improvement on the transmission capacity by using cooperative transmission.

\section{APPENDIX A}

Recall that we assume that simultaneous transmitters are modeled by homogeneous Poisson point process. The set $\Phi$ can be written as

$$
\Phi=\left\{X_{1}, X_{2}, \ldots\right\}=X_{n}
$$

Let $f(x)=P_{t}|x|^{-\alpha}$ be a measurable function of $\Phi$, the sum of $f(x)$ over $X_{i}$ in $\Phi$ can be written as

$$
\sum_{x \in \Phi} f(x)=\sum_{i \in \Phi} P_{t}\left|X_{i}\right|^{-\alpha}
$$

Since each $f(x)>0$, according to Campbell's theorem [10], for any non negative measurable function $f$, the calculation of mean value can be simplified as

$$
E\left(\sum_{x \in \Phi} f(x)\right)=\rho \int f(x) d x .
$$

where $\rho$ is the density of Poisson point process.

As can be seen in Fig. 3, for path loss model, we assume that no transmitter (Tx) is allowed within radius $s$. This is a sensible assumption, e.g., we may imagine the MAC protocol can prevent transmitting through scheduling within the radius $s$. All the transmitters (hollow circles) are poisson distributed in the path loss region.

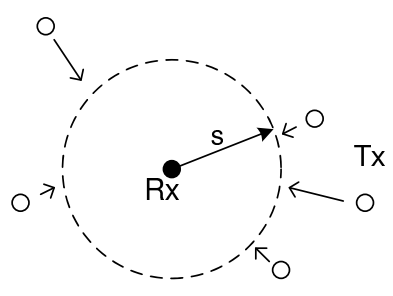

Fig. 3. Far-field assumption in wireless networks

Therefore, consider in a $2 \mathrm{D}$ network, we can derive the mean value of interference as

$$
\begin{aligned}
E\left(\sum_{x \in \Phi} f(x)\right) & =\rho \int f(x) d x \\
& =\rho P_{t} \int_{0}^{2 \pi} \int_{s}^{\infty} r^{-\alpha} r d \theta d r \\
& =2 \pi \rho P_{t} \int_{s}^{\infty} r^{(-\alpha+1)} d r \\
& =\frac{2 \pi P_{t} \rho s^{2-\alpha}}{\alpha-2}
\end{aligned}
$$

According to [11], the variance is derived as

$$
\begin{aligned}
\operatorname{Var}\left(\sum_{x \in \Phi} f(x)\right) & =\rho \int f^{2}(x) d x \\
& =\rho P_{t}^{2} \int_{0}^{2 \pi} \int_{s}^{\infty} r^{-2 \alpha} r d \theta d r \\
& =2 \pi \rho P_{t}^{2} \int_{s}^{\infty} r^{(-2 \alpha+1)} d r \\
& =\frac{\pi P_{t}^{2} \rho s^{2(1-\alpha)}}{\alpha-1} .
\end{aligned}
$$

\section{REFERENCES}

[1] J. N. Laneman and G. W. Wornell, "Distributed space-time-coded protocols for exploiting cooperative diversity in wireless networks," IEEE Trans. Information Theory, vol. 49, pp. 2415-2425, Oct. 2003.

[2] J. N. Laneman, D. N. Tse, and G. W. Wornell, "Cooperative diversity in wireless networks: Efficient protocols and outage behavior," IEEE Trans. On Information Theory, vol. 50(12), Dec. 2004.

[3] A. E. Khandani, "Cooperative routing in wireless networks," Ph.D. dissertation, MIT, 2004.

[4] Z. Sheng, Z. Ding, and K. K. Leung, "Interference subtraction with supplementary cooperation in wireless cooperative networks," in IEEE International Conference on Communications (ICC), Germany, 2009.

[5] P. Gupta and P. Kumar, "The capacity of wireless networks," IEEE Transactions on Information Theory, vol. 46, pp. 388-404, March 2000.

[6] S. P. Weber, X. Yang, J. G. Andrews, and G. de Veciana, "Transmission capacity of wireless ad hoc networks with outage constraints," IEEE Transactions on Information Theory, vol. 51, pp. 4091-4102, December 2005.

[7] S. Toumpis and A. J. Goldsmith, "Capacity regions for wireless ad hoc networks," IEEE Transactions on Wireless Communication, vol. 24, pp. 736-748, May 2003.

[8] J. Proakis, Digital Communication. McGraw-Hill, New York, 2000.

[9] N. Abramson, "The aloha system- another alternative for computer communication," in Proc. AFIPS, 1970.

[10] D. Stoyan, W. S. Kendall, and J. Mecke, Stochastic Geometry and Its Applications, second edition. John Wiley and Sons, 1995.

[11] R. A. DuBridge, "Campbell theorem - system concepts and results," IEEE Transactions on Nuclear Science, vol. 14, pp. 241-246, November 2007. 